\title{
ANÁLISIS DEL SISTEMA DE TRANSPORTE PÚBLICO Y LA CONTAMINACIÓN DEL AIRE DE LOS VEHÍCULOS LIVIANOS EN LA CIUDAD DE TACNA - 2014
}

\author{
Analysis of the public transport system and air pollution of light vehicles in the city of \\ Tacna - 2014
}

\section{Pablo Cesar Arcaya Pancca ${ }^{4}$}

\section{RESUMEN}

Objetivo: La presente investigación tiene como objetivo analizar el sistema de transporte público y la contaminación del aire de los vehículos livianos en la ciudad de Tacna - 2014.

Método: La investigación corresponde al tipo básica. El Diseño de Investigación es descriptivo correlacional. La metodología utilizada fue a través de la recopilación de información referente a la contaminación del aire, por parte de Gobierno Regional de Tacna, como es el proyecto de Monitoreo de la calidad del aire en la región de Tacna para verificar que los datos recopilados cumplen con los estándares de calidad ambiental y límites permisibles, así como la empresa Cortec S.R.L. Revisiones Técnicas para vehículos livianos en la ciudad de Tacna, para conocer qué cantidad de vehículos gasolineros y petroleros cumplen con las revisiones técnicas durante los meses del año 2014, así como encuestas efectuadas a la población para saber su opinión sobre la contaminación de los vehículos livianos.

Resultados: Los resultados obtenidos fueron que los valores de material partículado de $2.5\left(\mathrm{ug} / \mathrm{m}^{3}\right)$, sobrepasan los estándares de calidad ambiental (ECA), en los distritos de Alto de la Alianza (ISTP Francisco de Paula Gonzáles Vigil-Av. Jorge Basadre), Ciudad Nueva (Centro de Salud Ciudad Nueva-Calle Daniel Alcides Carrión) y Tacna (Gran Hotel Tacna-Av. Bolognesi),donde existen vehículos petroleros y antiguos que contaminan más el aire, así como hay desconocimiento de parte de las autoridades respecto a la contaminación del aire causada por los vehículos livianos.

Conclusión: Se llegó a la conclusión general de que se requiere tomar medidas que permitan reducir la contaminación del aire, y se cuente con información histórica de la contaminación frente al aumento del parque automotor.

Palabras claves: Parque automotor, contaminación de aire, Calidad de aire.

\section{ABSTRACT}

Objective: This research aims to analyze the system of public transport and air pollution light vehicles in the city of Tacna - 2014.

Method: The methodology used was through the collection of information on air pollution, by the Regional Government of Tacna, such as project monitoring air quality in the region of Tacna to verify that the data collected meet environmental quality standards and permissible limits and the company Cortec SRL Technical Reviews for light vehicles in the city of Tacna, to know how much of gasolineros and oil vehicles meet technical reviews during the months of 2014 and surveys the population to know your opinion on the contamination of light vehicles.

Results: The results were that the values of Particulate Matter 2.5 (ug / m3), exceed the environmental quality standards (ECA), in the districts of Alto de la Alianza (ISTP Francisco de Paula Gonzalez Vigil-Av Jorge Basadre. ), New Town (Health Centre New Town-Street Daniel Alcides Carrión) and Tacna (Gran Hotel Tacna Av. Bolognesi), where there are oil and old vehicles that pollute more air, and there is ignorance on the part of the authorities regarding air pollution caused by light vehicles.

Conclusion: It came to the general conclusion that is required to take measures to reduce air pollution, and have obtained historical information pollution versus increased fleet. Keywords: fleet, air pollution, air quality.

\footnotetext{
4 Magister en Ingeniería Civil con mención en Transporte
} 


\section{INTRODUCCIÓN}

Tacna es una de las ciudades en el país que tiene un alto crecimiento problacional y de vehículos que contaminan el aire y afectan la salud de la población a través de los gases tóxicos que emiten los vehículos petroleros y gasolineros, en las diferentes calles y avenidas de la ciudad de Tacna.

En los últimos años el parque automotor de Tacna ha ido en aumento, así como los diferentes tipos de vehículos de transporte público (como son los vehículos livianos), generando problemas de contaminación del aire. El problema fundamental respecto de la contaminación atmosférica es la ausencia de normatividad para aire limpio. El Perú no cuenta con normas o estándares de calidad del aire ni con estándares de emisión. El monitoreo que realiza el SENAMHI, pesa la carga de polvo atmosférico, pero no analiza sus componentes. De otro lado, el Código del Medio Ambiente y Recursos Naturales no contiene disposiciones relativas a la contaminación atmosférica. La Asociación Peruana Contra la Contaminación Atmosférica tiene una propuesta de normas de calidad del aire y ha participado activamente en un proyecto de reglamento para el control de la contaminación atmosférica que fuera coordinado por DIGESA y el Ministerio de Industria, Comercio, Turismo e Integración en 1992.

La contaminación del aire se inició con el descubrimiento del fuego; se incrementó con los procesos de la industrialización. La revolución industrial utilizó como combustible la leña para la generación de vapor, energía para la tecnología del hierro y acero. Durante el siglo XVI, en Inglaterra se utilizaban la madera como combustible, generando su escases, siendo sustituida por la hulla como combustible, la misma que libera gran cantidad de sustancias químicas al ambiente como azufre, nitrógeno etc.

En los años 1837 a 1901, Inglaterra, sufrió los efectos de la contaminación industrial, manifestándose casos de enfermedades desconocidas atribuidas como transmisibles. En el siglo XVIII comenzaron a extenderse las áreas urbanas, aumentó la dependencia de las ciudades hacia el campo para la obtención de los alimentos. El desarrollo industrial aceleró la emisión de contaminantes atmosféricos y partículas, durante el siglo XIX la contaminación atmosférica causada por la industria se identificó como un problema, para la salud.

En el año 1952, Londres fue invadida por una niebla asociada a un régimen anticiclónico y a una inversión térmica; como resultado de las bajas temperaturas los calefactores estuvieron funcionando y las condiciones meteorológicas hicieron que la nube de humo persistiera sobre la ciudad durante varios días; causó 2851 muertes en solo 9 días y 1225 fallecimientos a la semana siguiente. En 1956 la niebla que envolvió a la ciudad durante 18 horas causó 1000 muertes más de las previstas. En 1889, se convocó la convención internacional para la prohibición de las armas químicas.

Durante la guerra mundial (1915), Alemania utilizó por primera vez el cloro elemental, que desencadenó una intensa guerra química en el mundo. En 1972, en Estocolmo, se celebró la primera conferencia sobre el ambiente humano de la organización de naciones unidas, movimientos públicos persuadieron a los gobiernos a desarrollar una legislación para limitar las emisiones de contaminantes químicos tóxicos al ambiente. En el 1982, se creó la comisión mundial de ambiente y desarrollo. En 1983, bajo la presidencia en esta comisión de la Dra. Gro Harlem Brundtland, primera ministra de Noruega, se intensificó el trabajo relacionado con temas del ambiente y la salud.

En el informe "nuestro futuro común", donde se estableció pautas fundamentales en los problemas ambientales se hizo un llamado al "desarrollo sostenible", para satisfacer las necesidades de las generaciones presentes sin comprometer la satisfacción de las 
necesidades de las generaciones futuras. El creciente desarrollo económico y tecnológico comenzó a borrar la línea divisoria entre los problemas ambientales locales y globales, entre los que se destacan la acidificación del ambiente, la destrucción de la capa de ozono y el incremento del calentamiento global de la tierra.

A consecuencia de ello y como una respuesta a la preocupación sobre la contaminación mundial y en una misión compartida: En 1982, se celebró la conferencia multilateral sobre la acidificación del ambiente. Las precipitaciones ácidas tienen un $\mathrm{PH}$ inferior a 5.0 y sus precursores son el bióxido de azufre y los óxidos de nitrógeno causado por la quema de combustibles fósiles. Estos gases oxidados en la atmósfera, reaccionan con el agua de lluvia formando ácidos sulfúrico y nítrico; fenómeno que daña la flora y la fauna, la pérdida del patrimonio cultural, por la destrucción de monumentos históricos y zonas arqueológicas (Valencia, 2009).

En 1985, se aprobó el convenio de Viena para la protección de la capa de ozono, auspiciado por las naciones unidas, que adoptó medidas para proteger la salud y el ambiente de los efectos que provoca el agotamiento del ozono estratosférico, 49 países acordaron proteger la capa de ozono. Los compuestos químicos como los Clorofluorocarbonados (CFC), que se encuentran en los aerosoles, los gases refrigerantes y los halones que son compuestos formados por bromo, flúor y carbono, son sustancias que se caracterizan por tener una gran capacidad de dañar la capa de ozono.

En 1987, se firmó el protocolo de Montreal para fijar límites a la producción de CFC y halones, el cual entró en vigor en 1989 y ha sufrido sucesivas enmiendas. Se estima que solo los países subdesarrollados utilizan el $16 \%$ del consumo mundial y resulta necesaria la transferencia de tecnologías para dejar de utilizar dichos gases. En 1992, se realizaron cumbres mundiales sobre medio ambiente y desarrollo sostenible en Río de Janeiro y Johannesburgo (2002), los que han evidenciado la necesidad de mejorar la calidad del aire y alcanzar un desarrollo social donde prevalezca la equidad y el respeto por la naturaleza. En 1985, la organización meteorológica mundial (OMM) y el Programa de las Naciones Unidas para el medio ambiente (PNUMA) invitaron a científicos de 29 países para analizar el calentamiento de la atmósfera, se creó un comité técnico para estudiar este fenómeno. Durante 1990, la asamblea general de las naciones unidas creó el comité intergubernamental de negociación para la convención marco sobre el cambio climático (CIN/CMCC), con representantes de 150 países.

El aire es el elemento básico de todo ser vivo. Diariamente nuestros pulmones filtran unos 15 kilómetros de aire atmosférico, mientras que sólo absorbemos 25 kilos de agua y menos de 1.5 kilos de alimentos. El aire está compuesto por una mezcla de gases que constituye la atmósfera: nitrógeno, oxígeno, argón, anhídrido carbónico; además contiene vapor de agua y gases raros menores, como el helio, criptón, radón, xenón y ozono. La contaminación del aire es la presencia de sustancia o elemento que en determinados niveles de concentración en el aire genera riesgos a la salud y al bienestar humano. Su presencia en el aire obedece a emisiones naturales y a las emisiones procedentes de las diversas actividades del hombre. Los contaminantes de la atmósfera pueden ser:

- Químicos: constituidos por sustancias de diverso estado físico, se originan en los múltiples procesos de transformación de la materia. pueden estar en estado gaseoso o particulado.

- Físicos: son formas de energía muy difundidos en las variadas actividades de la sociedad contemporánea. ejemplo: el ruido.

- Biológicos: constituidos por los organismos vivientes que arriban a la atmósfera en formas muy diversas como el polen y esporas, mohos, hongos, virus, etc. 
Las fuentes de contaminación atmosférica en el país son:

- $\quad$ Las industrias minero-metalúrgicas, que incluyen las fábricas de cemento y la industria del petróleo y sus derivados.

- $\quad$ Parque automotor

- $\quad$ Fábricas de harina de pescado.

- Industria textil.

- Industria alimentaria.

- Industria química.

- $\quad$ Las malas costumbres de la población (quema de basuras y desmonte).

Las consecuencias de la contaminación atmosférica son a largo plazo y difíciles de determinar, sin embargo estudios de probabilidad permiten señalar que determinados agentes son factores de riesgo ambiental y de enfermedad. Entre las enfermedades que producen los contaminantes químicos se encuentran el cáncer a la piel, las "cataratas" de los ojos, la arterioesclerosis, la alteración de la inmunidad y el menoscabo de la inteligencia a causa de la exposición al plomo. La relación de normas legales que se detallan posteriormente se ocupa de los contaminantes que afecten nuestra atmósfera.

Debemos resaltar que en nuestro país se han establecido hasta la fecha los límites permisibles de contaminación atmosférica, utilizándose para los estudios, los parámetros internacionales. Sin embargo, existen patrones nacionales de calidad del aire, aprobado por diferentes entes del sector público y privado, pero que lamentablemente no se difunde.

Las emisiones tóxicas se clasifican en reglamentadas y no reglamentadas. su acción sobre el organismo humano es diferente: desde sensaciones desagradables hasta enfermedades graves, incluyendo el cáncer, en concentraciones considerables pueden tener efecto letal. Las emisiones tóxicas se refieren a: $\mathrm{CO}, \mathrm{CXHY}, \mathrm{NOX}$, el humeado (humo) y el hollín, así como partículas sólidas (PS).

A continuación, se muestran los factores que determinan la contaminación del aire en grandes ciudades:

- El uso masivo del automóvil .

- Déficit cualitativo y cuantitativo en el transporte público de pasajeros.

- Falta de adecuación tecnológica para el uso de combustibles menos contaminantes y dispositivos de reducción de la contaminación.

- $\quad$ Escaso mantenimiento del parque automotor de vehículos.

- Congestión vehicular.

- Desactualización normativa vigente y falta de adecuación de los estándares de emisión.

- Deficiencia de sistemas de controles de funcionamiento vehicular.

- $\quad$ Falta de programas de educación ambiental y concientización sobre las implicancias para la salud de la contaminación del aire.

Los distintos tipos de vehículos según su peso y potencia, se mueven de diferente forma en las vías. Además, poseen diferentes configuraciones de motores y tecnologías, y por ende, aportan en forma distinta al inventario de emisiones de contaminantes. Las categorías se muestran a continuación:

- Buses de servicio local.

- Buses de servicio interprovincial.

- Camiones livianos, medianos y pesados.

- Automóviles particulares. 
- Automóviles taxis.

- Camionetas.

- Motocicletas.

El monóxido de carbono (CO) es un gas tóxico producido en la combustión incompleta en base a carbono en ambientes con poca presencia de oxígeno. Puesto que el gas es inodoro, insípido e incoloro, a menudo no hay advertencia en las personas sobre la exposición a este gas. El monóxido de carbono sobrevive en la atmósfera típicamente por cuatro semanas, tiempo durante el cual se oxida gradualmente y forma dióxido de carbono. En los motores de combustión interna se obtiene como resultado de la mezcla heterogénea del combustible con el oxígeno. Se ha avanzado mucho en la reducción de este contaminante gracias a un mejor control de la dosificación de combustible así como también el uso de catalizadores de tres vías, que continúan con la oxidación del $\mathrm{CO}$ a $\mathrm{CO}_{2}$ reduciendo en más de un $90 \%$ las emisiones de este gas. La preocupación principal por la contaminación del CO está en áreas urbanas, particularmente en la exposición de conductores, ciclistas y peatones a altas concentraciones muy localizadas producto de congestión en vías urbanas.

El CO es uno de los agentes contaminantes más peligrosos para la salud humana porque causa en los seres humanos una rápida reducción en la capacidad de la sangre de transportar oxígeno, dando por resultado dolores de cabeza, fatiga, problemas respiratorios y en algunos casos la muerte por asfixia. El CO predomina en el gas de emisión, generado en la ciudad de Tacna superior al 2,00\%6,7. Es un gas incoloro, inodoro, más pesado que el aire, no tóxico. El porcentaje elevado de $\mathrm{CO}_{2}$ en la atmósfera produce el latido acelerado del corazón. Actualmente el contenido de este gas en la atmósfera alcanza 350 ppm aumentándose cada año en $0.5 \%$.

La particularidad de $\mathrm{CO}_{2}$, es que al subir en las capas superiores de la atmósfera absorbe intensivamente la radiación infrarroja reflejada por la superficie terrestre. Este fenómeno conduce al aumento de la temperatura promedio de la tierra, al que se le ha denominado " efecto invernadero", por eso en muchos países existen los programas especiales que prescriben, utilizando todos los medios posibles para contribuir a la disminución de las emisiones de $\mathrm{CO}_{2}$. Los daños de la contaminación del aire pueden ser:

- Daños a la vegetación: alteraciones foliares, reducción del crecimiento de las plantas, disminución de la floración, etc.

- $\quad$ Alteraciones del medio ambiente: reducción de la visibilidad, efecto de invernadero, afectación de la capa de ozono, lluvia ácida, etcétera.

- $\quad$ Efectos psicológicos sobre el hombre.

- $\quad$ Efectos fisiológicos sobre el hombre: agudos y crónicos.

- $\quad$ Pérdidas por efectos directos o indirectos en el ganado y en las plantas.

- $\quad$ Pérdidas por la corrosión de materiales y de sus revestimientos de protección.

- $\quad$ Pérdidas por gastos de mantenimiento de las edificaciones y la depreciación de objetos y mercancías expuestos.

- Gastos directos por la aplicación de medidas para reducir el humo y las emanaciones de las fábricas.

- Pérdidas indirectas por mayores gastos de transporte en tiempo de niebla contaminada, o de electricidad por la necesidad de encender el alumbrado antes del horario establecido.

- $\quad$ Gastos administrativos de la lucha contra la contaminación.

- $\quad$ Costo en investigaciones destinadas a la contaminación ambiental.

Para la prevención y control de la contaminación del aire, se pueden considerar los siguientes: 
- $\quad$ Aplicación de las medidas normativas de la calidad del aire. Planificación urbana y regional.

- $\quad$ Reducción de la generación de contaminantes.

- Control de emisión de partículas en la fuente, con cámaras de sedimentación, separador inercial, purificación por vía húmeda, filtración y precipitación electrostática; control de las emisiones gaseosas por combustión, absorción o adsorción.

Las principales características en relación a la vialidad de la ciudad de Tacna son los siguientes:

- $\quad$ El sistema vial de la ciudad de Tacna ocupa un área de 1485 has. que representa aproximadamente el $35 \%$ del área urbana actual. en general las vías deben representar del $45 \%$ al $55 \%$ del área de una ciudad para brindar un buen servicio (ver anexo 07 ).

- Sobresaturación vehicular de vías principales del casco urbano central de la ciudad (ver anexo 08).

- $\quad$ Por parte del transporte público y privado; especialmente en horas punta (de 6:30 a.m. a 8:30 a.m., de 12 m. a 1:30 p.m. y de 6:00 p.m. a 7:30 p.m.).

- $\quad$ Congestionamiento vehicular de la zona monumental en las horas punta.

- Existencia de intersecciones viales críticas, principalmente en los óvalos y las actuales intersecciones semaforizadas, e insuficiente señalización vial (vertical y horizontal).

- Desarticulación vial transversal de la ciudad, que no permite una circulación adecuada de flujos de transporte norte - sur.

El sistema vial provincial en Tacna está formada por una red vial que satisface la demanda de transporte actual y futura, y que garantiza la interrelación entre las diferentes áreas de la provincia de Tacna, así como sus vinculaciones con el resto del país. El sistema vial provincial es un elemento estratégico que estructura el modelo de acondicionamiento territorial que el presente plan propone, y permitirá orientar las acciones y proyectos para el reforzamiento de los ejes de desarrollo; y la vinculación de las áreas de especialización propuestas y los centros poblados para una mejor articulación y desempeño de sus roles y funciones.

Se muestra a continuación la siguiente clasificación funcional de vías:

\section{a) Vías nacionales}

Son aquellas vías que permiten la integración y articulación de la provincia de Tacna con el resto del país, y con los países vecinos en este caso especifico el Chile.

b) Vías regionales

Son aquellas vías que permiten la articulación provincial y regional; constituyendo una alternativa complementaria para la mejor integración de la provincia de Tacna con el resto del país, a través de la conexión de éstas a otras vías regionales y nacionales.

c) Vías departamentales

Son aquellas que permiten la interconexión de la provincia de Tacna con las otras provincias del departamento; teniendo por tanto un ámbito departamental.

d) Vías vecinales

Son aquellas vías que permiten la articulación de centros poblados menores de la provincia, siendo su ámbito de nivel provincial.

Los transportes en la ciudad de Tacna se organizan en terrestre, ferroviario y aéreo con las siguientes características:

El transporte terrestre en la ciudad de Tacna, sufre un caos debido a su gran dinámica y al tamaño de su parque automotor, el mismo que al año 2000 está compuesto por 37,120 
vehículos; lo que determina una tasa de motorización de 1 vehículo por cada 6 personas, cercana a la tasa de motorización del área metropolitana Lima-Callao que es de 1 vehículo por cada 5 personas. Se organiza a su vez en transporte urbano, interurbano interdepartamental e internacional. Los flujos de transporte provincial se dan con mayor intensidad a través de la carretera panamericana, al igual que el intercambio, del mismo modo el intercambio interprovincial con las otras provincias, las cuales tienen además alternativamente facilidades mayores, desde Sama tanto por la panamericana como por la costanera asimismo bajando por Tarata hacia Tacna.

Por otro lado el intercambio internacional, genera un intenso flujo de vehículos por la carretera panamericana desde Arica. El sistema de transportes en la provincia de Tacna se puede clasificar en tres tipos: transporte urbano, interurbano e internacional.

\section{MATERIALES Y MÉTODOS}

La presente investigación es no experimental, porque estaba orientado al análisis del sistema de transporte público y la contaminación del aire generada por los vehículos livianos en la ciudad de Tacna. El nivel de investigación fue descriptivo correlacional por que está orientado a medir las características de la contaminación del aire generada por los vehículos livianos en la ciudad de Tacna. Fue correlacional, porque evaluó la variable contaminación del aire generada por los vehículos livianos, el cual dependió del tipo de combustible usado, año de fabricación del vehículo, velocidad del viento y otros. Las encuestas se realizaron a la población en nuestra ciudad de Tacna. Las encuestas se aplicarán a una muestra probabilística aleatoria simple de la población. El tiempo corresponde al año 2014, durante los meses de febrero a diciembre.

La presente investigación comprendió únicamente lo referente a la situación de la contaminación del aire de la ciudad de Tacna, provocados por los vehículos livianos en el año 2014. El área de influencia de la investigación, comprendió los distritos de: Tacna, Gregorio Albarracín Lanchipa, Ciudad Nueva, Alto de la Alianza y Pocollay. La población está conformada por 40,778 unidades livianas registradas en Tacna, el año 2014. La información obtenida de la base de datos del Ministerio de Transportes y Comunicaciones-Provias nacional. La muestra estuvo constituida por 8,311 unidades livianas que acuden a la revisión de humos tóxicos a la planta de control de humos, la misma que es determinada por los índices de cálculo muestral con $5 \%$ de error. Una vez determinado el tamaño de la muestra se seleccionará la muestra a través de una selección determinada a discreción del investigador dentro de la ciudad de Tacna.

Se sistematizo la información de acuerdo a la formulación del problema, de los objetivos y la hipótesis de la investigación. El procesamiento de datos a partir de la toma de información en campo, se proceso la información en el paquete estadístico SPSS y, para su análisis se utilizo la estadística descriptiva, así mismo los resultados se presentaron en tablas, figuras y gráficos. Se realizo la revisión de información de libros, artículos de publicaciones, relacionadas al tema de la tesis. 


\section{RESULTADOS}

A continuación se muestra el control de emisión de gases en vehículos para gases $\mathrm{CO}, \mathrm{CO}_{2}$, $\mathrm{O}_{2}$. Los resultados del control de humos de Cortec S.R.L., se presentan en la tabla siguiente:

Tabla 1: Revisiones técnicas de humos en vehículos (Enero a diciembre 2014)

\begin{tabular}{|c|c|c|c|c|c|c|}
\hline \multirow[t]{2}{*}{$\mathrm{N}^{\circ}$} & \multirow[t]{2}{*}{ DESCRIPCIÓN } & \multirow[t]{2}{*}{ CANTIDAD } & \multicolumn{4}{|c|}{$\begin{array}{r}\text { REVISIONES } \\
\text { TECNICAS HUMOS } \\
\end{array}$} \\
\hline & & & $\begin{array}{c}\text { SI } \\
\text { CUMPLE }\end{array}$ & $\%$ & $\begin{array}{c}\text { NO } \\
\text { CUMPLE }\end{array}$ & $\%$ \\
\hline 1 & AUTOMOVILES & 4066 & 4046 & $99.50 \%$ & 20 & $0.50 \%$ \\
\hline 2 & STATION WAGON & 4153 & 4128 & $99.40 \%$ & 25 & $0.60 \%$ \\
\hline 3 & CAMIONETA PICK UP & 29 & 28 & $95.00 \%$ & 1 & $5.00 \%$ \\
\hline 4 & CAMIONETA RURAL & 15 & 12 & $79.00 \%$ & 3 & $21.00 \%$ \\
\hline 5 & CAMIONETA PANEL & 5 & 4 & $88.00 \%$ & 1 & $12.00 \%$ \\
\hline 6 & OMNIBUS & 8 & 7 & $85.00 \%$ & 1 & $15.00 \%$ \\
\hline 7 & CAMION & 13 & 11 & $87.00 \%$ & 2 & $13.00 \%$ \\
\hline 8 & $\begin{array}{l}\text { REMOLCADOR } \\
\text { REMOLQUE Y }\end{array}$ & 12 & 9 & $78.00 \%$ & 3 & $22.00 \%$ \\
\hline 9 & SEMIREMOLQUE & 10 & 8 & $77.00 \%$ & 2 & $23.00 \%$ \\
\hline & TOTAL & 8311 & 8253 & $99.30 \%$ & 58 & $0.70 \%$ \\
\hline
\end{tabular}

Fuente: Cortec S.R.L. revisiones técnicas, 2014.

\section{Análisis}

De la tabla 1 se resume que del $100 \%$ de vehículos que pasaron las revisiones técnicas de humos, durante los meses de enero a diciembre del 2014 , el $0.70 \%$ no cumplen con las exigencias técnicas del control de humos. Lo cual significa que existe responsabilidad en los propietarios por mantener a los vehículos en revisiones permanentes para evitar ser excluido del parque automotor por no reunir los estándares que preservan la calidad del aire.

Tabla 2: Ubicación de puntos de monitoreo.

\begin{tabular}{|c|c|c|c|c|c|}
\hline $\mathbf{N}^{\circ}$ & \multirow{2}{*}{ 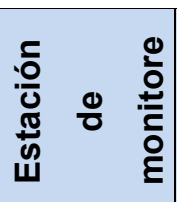 } & \multirow[t]{2}{*}{ Descripción } & \multicolumn{2}{|c|}{ Coordenadas UTM } & \multirow{2}{*}{$\begin{array}{l}\text { Altura } \\
\text { (msnm) }\end{array}$} \\
\hline Ítem & & & Este & Norte & \\
\hline 1 & $\mathrm{E}-01$ & Centro de ESSALUD & 367377 & 8004944 & 514 \\
\hline 2 & $\mathrm{E}-02$ & Centro de Postgrado de la UNJBG & 368814 & 8008580 & 591 \\
\hline 3 & $\mathrm{E}-03$ & $\begin{array}{l}\text { I.S.T.P. Francisco de Paula Gonzales } \\
\text { Vigil }\end{array}$ & 368367 & 8010120 & 618 \\
\hline 4 & E-04 & C.S. Ciudad Nueva & 369279 & 8011078 & 668 \\
\hline 5 & E-05 & Gran Hotel Tacna & 367779 & 8007797 & 567 \\
\hline 6 & E-06 & Cuartel de la Policía Militar & 368348 & 8008532 & 594 \\
\hline 7 & E-07 & Ciudad Universitaria de la U.N.J.B.G. & 367784 & 8006750 & 545 \\
\hline
\end{tabular}

Fuente: Monitoreo de la Calidad del aire-Gob. Regional Tacna, 2014. 
De los cuadros anteriores se resume que:

- ESSALUD Gregorio Albarracín (distrito Gregorio Albarracin) (E-01): se encuentran dentro de los estándares de calidad ambiental (ECA), cumple con los valores máximos permisibles.

- Centro de Postgrado UNJBG (E-02): se encuentran dentro de los estándares de calidad ambiental (ECA), es decir cumple con los valores máximos permisibles.

- ISTP Francisco de Paula González Vigil (distrito Alto de la Alianza) (E-03): las concentraciones de $\mathrm{PM}_{2.5}$, sobrepasa los estándares de calidad ambiental (ECA) de 25 $\mathrm{ug} / \mathrm{m}^{3}$ en 1 día de muestreo de acuerdo al D.S. 003-2008-MINAM - Estándares Nacionales de Calidad Ambiental (ECA) para Aire.

- Centro de Salud Ciudad Nueva (distrito de Ciudad Nueva) (E-04): se encuentran dentro de los estándares de calidad ambiental (ECA) de $25 \mathrm{ug} / \mathrm{m}^{3}$ en 1 día de muestreo de acuerdo al D.S. 003-2008-MINAM - Estándares Nacionales de Calidad Ambiental (ECA) para Aire.

- Gran Hotel Tacna (distrito de Tacna-avenida Bolognesi) (E-05): las concentraciones de $\mathrm{PM}_{2.5}$, sobrepasa los estándares de calidad ambiental (ECA) de $25 \mathrm{ug} / \mathrm{m}^{3}$ en 1 día de muestreo de acuerdo al D.S. 003-2008-MINAM - Estándares Nacionales de Calidad Ambiental (ECA) para Aire.

- Policía militar cercado (distrito de Tacna) (E-06): las concentraciones de PM 2.5 , sobrepasa los estándares de calidad ambiental (ECA) de $25 \mathrm{ug} / \mathrm{m}^{3}$ en 1 día de muestreo de acuerdo al D.S. 003-2008-MINAM - Estándares Nacionales de Calidad Ambiental (ECA) para Aire.

- Ciudad Universitaria UNJBG (distrito de Tacna) (E-07): las concentraciones de PM 2.5 , sobrepasa los estándares de calidad ambiental (ECA) de $25 \mathrm{ug} / \mathrm{m}^{3}$ en 1 día de muestreo de acuerdo al D.S. 003-2008-MINAM - Estándares Nacionales de Calidad Ambiental (ECA) para Aire. 


\section{Resultado-Objetivo 01:}

Tabla 3: Grado de conocimiento que tiene la población sobre el actual sistema de transporte público y la contaminación atmosférica de los vehículos livianos en la ciudad de Tacna.

\begin{tabular}{|c|c|c|c|c|c|c|c|}
\hline $\mathrm{N}^{\circ}$ & RESULTADOS & FAVORABLE & $\%$ & $\begin{array}{c}\text { NO } \\
\text { FAVORABLE }\end{array}$ & $\%$ & TOTAL & unidad \\
\hline 1 & $\begin{array}{l}\text { Si } \\
\text { contaminación anoce la } \\
\text { causada por los } \\
\text { livianos }\end{array}$ & 29 & $58.00 \%$ & 21 & $42.00 \%$ & 50 & personas \\
\hline 2 & $\begin{array}{l}\text { No existen estudios } \\
\text { suficientes y efectivos de la } \\
\text { contaminación de los } \\
\text { vehículos livianos }\end{array}$ & 37 & $74.00 \%$ & 13 & $26.00 \%$ & 50 & personas \\
\hline 3 & sistema respiratorio & 27 & $54.00 \%$ & 23 & $46.00 \%$ & 50 & personas \\
\hline 4 & $\begin{array}{l}\text { gases tóxicos que emanan } \\
\text { los vehículos livianos - } \\
\text { manos o menos }\end{array}$ & 26 & $52.00 \%$ & 24 & $48.00 \%$ & 50 & personas \\
\hline 5 & $\begin{array}{l}\text { la mayor contaminación del } \\
\text { aire por gases tóxicos en } \\
\text { avenida Bolognesi }\end{array}$ & 29 & $58.00 \%$ & 21 & $42.00 \%$ & 50 & personas \\
\hline 6 & $\begin{array}{l}\text { Nunca ha observado } \\
\text { autoridades encargadas en } \\
\text { la prevención de la } \\
\text { contaminación del aire }\end{array}$ & 40 & $80.00 \%$ & 10 & $20.00 \%$ & 50 & personas \\
\hline 7 & $\begin{array}{l}\text { Vehículos de muchos años } \\
\text { de antigüedad contaminan } \\
\text { más el aire }\end{array}$ & 34 & $68.00 \%$ & 16 & $32.00 \%$ & 50 & personas \\
\hline 8 & $\begin{array}{l}\text { Más o menos las } \\
\text { autoridades y funcionarios } \\
\text { conocen sobre el transporte } \\
\text { público y la contaminación } \\
\text { ambiental de los vehículos } \\
\text { livianos }\end{array}$ & 19 & $38.00 \%$ & 31 & $62.00 \%$ & 50 & personas \\
\hline 9 & \begin{tabular}{llrr} 
Más de & $10 \quad$ años de \\
antigüedad & \multicolumn{2}{c}{ existen } \\
vehículos & livianos & por \\
donde vive & & & \\
\end{tabular} & 35 & $70.00 \%$ & 15 & $30.00 \%$ & 50 & personas \\
\hline & promedio & 31 & $62.00 \%$ & 19 & $38.00 \%$ & 50 & personas \\
\hline
\end{tabular}

Fuente: Elaboración propia.

Según los resultados obtenidos el grado de conocimiento de la población sobre el actual sistema de transporte público y la contaminación atmosférica de los vehículos livianos en la ciudad de Tacna es inadecuado.

\section{Resultado-Objetivo 02:}

Recopilar datos obtenidos en campo de la contaminación atmosférica de los vehículos livianos en la ciudad de Tacna del sistema de transporte público para los Límites Máximos Permisibles (LMP) y Estándares de Calidad Ambiental (ECA).

Se obtuvieron resultados del Gobierno Regional de Tacna, así como de Cortec S.R.L., los resultados se muestran en la tabla 4. 
Tabla 4: Resultados del análisis del monitoreo de la calidad de aire - Gobierno Regional de Tacna

\begin{tabular}{|c|c|c|c|c|c|c|c|c|}
\hline $\mathrm{N}^{\circ}$ & & FAVORABLE & $\%$ & $\begin{array}{c}\text { NO } \\
\text { FAVORABLE }\end{array}$ & $\%$ & TOTAL & UNIDAD & OBSERVACION \\
\hline 1 & $\begin{array}{l}\text { ESSALUD Gregorio } \\
\text { Albarracin (distrito } \\
\text { Gregorio Albarracin) } \\
(\text { E-01) }\end{array}$ & 3 & $100.00 \%$ & 0 & $0.00 \%$ & 3 & datos & $\begin{array}{l}\text { SI CUMPLE CON } \\
\text { LOS ECAS }\end{array}$ \\
\hline 2 & $\begin{array}{l}\text { Centro de Postgrado } \\
\text { UNJBG (distrito de } \\
\text { Tacna)(E-02) }\end{array}$ & 4 & $100.00 \%$ & 0 & $0.00 \%$ & 4 & datos & $\begin{array}{l}\text { SI CUMPLE CON } \\
\text { LOS ECAS }\end{array}$ \\
\hline 3 & $\begin{array}{l}\text { ISTP Francisco de } \\
\text { Paula Gonzáles Vigil } \\
\text { (distrito Alto de la } \\
\text { Alianza) (E-03) }\end{array}$ & 3 & $75.00 \%$ & 1 & $25.00 \%$ & 4 & datos & $\begin{array}{l}\text { SI CUMPLE CON } \\
\text { LOS ECAS }\end{array}$ \\
\hline 4 & $\begin{array}{l}\text { Centro de Salud } \\
\text { Ciudad Nueva (distrito } \\
\text { de Ciudad Nueva) (E- } \\
\text { 04) }\end{array}$ & 0 & $0.00 \%$ & 3 & $100.00 \%$ & 3 & datos & $\begin{array}{l}\text { SI CUMPLE CON } \\
\text { LOS ECAS }\end{array}$ \\
\hline 5 & $\begin{array}{l}\text { Gran Hotel Tacna } \\
\text { (distrito de Tacna- } \\
\text { Avenida Bolognesi) } \\
\text { (E-05) }\end{array}$ & 0 & $0.00 \%$ & 4 & $100.00 \%$ & 4 & datos & $\begin{array}{l}\text { NO CUMPLE CON } \\
\text { LOS ECAS }\end{array}$ \\
\hline 6 & $\begin{array}{l}\text { Policía militar } \\
\text { cercado } \\
\text { (distrito de } \\
\text { Tacna) (E-06) }\end{array}$ & 4 & $66.67 \%$ & 2 & $33.33 \%$ & 6 & datos & $\begin{array}{l}\text { NO CUMPLE CON } \\
\text { LOS ECAS }\end{array}$ \\
\hline 7 & $\begin{array}{l}\text { Ciudad } \\
\text { Universitaria } \\
\text { UNJBG } \\
\text { (distrito de } \\
\text { Tacna) (E-07) }\end{array}$ & 4 & $100.00 \%$ & 0 & $0.00 \%$ & 4 & datos & $\begin{array}{l}\text { NO CUMPLE CON } \\
\text { LOS ECAS }\end{array}$ \\
\hline
\end{tabular}

Fuente: Elaboración propia.

Según los resultados de la tabla 4 obtenidos de los puntos de monitoreo (E-05 al E-07), los datos obtenidos en campo con los Límites Máximos Permisibles no cumplen con todos los Estándares de Calidad Ambiental (ECA).

Tabla 5: Resultados de análisis de CORTEC, FUENTE EMISORA: Medición de humos con equipos en vehículos (tubo de escape).

\begin{tabular}{|c|c|c|c|c|c|c|c|c|c|c|}
\hline \multirow{3}{*}{$\mathrm{N}^{\circ}$} & \multirow{3}{*}{$\begin{array}{l}\text { VEHICULO (SEGÚN } \\
\text { TIPO DE } \\
\text { COMBUSTIBLE) }\end{array}$} & \multirow{3}{*}{ CANTIDAD } & \multirow{2}{*}{\multicolumn{2}{|c|}{$\begin{array}{l}\text { FUENTE DE } \\
\text { EMISION CO, } \\
\text { CO2 Y O2 }\end{array}$}} & \multicolumn{6}{|c|}{$\begin{array}{l}\text { LIMITES MAXIMOS PERMISIBLES } \\
(*)\end{array}$} \\
\hline & & & & & \multicolumn{3}{|c|}{ HASTA 1995} & \multicolumn{3}{|c|}{$\begin{array}{c}1996 \text { EN } \\
\text { ADELANTE }\end{array}$} \\
\hline & & & $\begin{array}{c}\text { SI } \\
\text { CUMPLE } \\
\end{array}$ & $\begin{array}{c}\text { NO } \\
\text { CUMPLE } \\
\end{array}$ & $\begin{array}{l}\text { CO ( } \% \\
\text { VOL.) } \\
\end{array}$ & $\begin{array}{r}\mathrm{HC} \\
(\mathrm{ppm}) \\
\end{array}$ & $\begin{array}{l}\mathrm{CO}+\mathrm{CO} 2 \\
(\% \mathrm{VOL} .) \\
\end{array}$ & \begin{tabular}{|c|} 
CO \\
$(\%$ \\
VOL.) \\
\end{tabular} & $\begin{array}{c}\mathrm{HC} \\
(\mathrm{ppm}) \\
\end{array}$ & $\begin{array}{c}\mathrm{CO}+\mathrm{CO} \\
2(\% \\
\text { VOL.) } \\
\end{array}$ \\
\hline 1 & \begin{tabular}{|l|} 
DIESEL \\
(PETROLEO) \\
\end{tabular} & 7615 & 6190 & 47 & 4.5 & 600 & 10 & 3.5 & 400 & 10 \\
\hline 2 & GASOLINEROS & 696 & 2043 & 31 & 4.5 & 600 & 10 & 3.5 & 400 & 10 \\
\hline & TOTAL & 8311 & 8233 & 78 & & & & & & \\
\hline
\end{tabular}

Fuente: Elaboración propia.

$\left(^{*}\right)$ Decreto supremo $\mathrm{N}^{\circ}$ 047-2001-MTC- LIMITES MAXIMOS PERMISIBLES 
De los resultados obtenidos, se aprecia que existen 8,233 vehículos que cumplen las revisiones técnicas de límites máximos permisibles, de la muestra de 8,311 vehículos.

\section{PRUEBA ESTADÍSTICA}

La prueba estadística fue el método de relación de Pearson (para las variables evaluación de transporte público ( Vehículos evaluados) y la contaminación ambiental ( si cumplen los estándares de humos).

Tabla 6: Vehículos evaluados versus resultados de monóxido de carbono.

\begin{tabular}{|l|c|c|c|}
\hline \multicolumn{2}{|c|}{$\begin{array}{c}\text { FLOTA VEHICULAR (REVISIONES } \\
\text { TECNICAS) }\end{array}$} & \multicolumn{2}{c|}{$\begin{array}{c}\text { MONOXIDO DE } \\
\text { CARBONO }\end{array}$} \\
\hline $\begin{array}{l}\text { TIPO DE COMBUST DE } \\
\text { VEHICULO }\end{array}$ & Evaluados & Si cumple & No cumple \\
\hline DIESEL(PETROLEO) & 7615 & 6190 & 47 \\
\hline GASOLINEROS & 696 & 2043 & 31 \\
\hline TOTAL & 8311 & 8233 & 78 \\
\hline
\end{tabular}

Fuente: Cortec S.R.L.- revisiones técnicas (2014).

\section{A: $\quad$ Formulación de las Hipótesis}

$\mathrm{H}_{0} ; \quad$ No existe relación entre la evaluación de transporte público y contaminación del ambientes.

$\mathrm{H}_{1}$; Si existe relación entre la evaluación de transporte público y contaminación del ambiente.

\section{B: Determinación del tipo de correlación}

Como las variables en estudio son cuantitativas, el Análisis de correlación pertinente es el de Karl Pearson ( $r$ )

\section{C: Determinación de fórmula de correlación}

Fórmula con datos discretos

$$
r=\frac{N \cdot\left(\sum X . Y\right)-\left(\sum X\right) \cdot\left(\sum Y\right)}{\sqrt{\left[N\left(\sum X^{2}\right)-\left(\sum X\right)^{2}\right]\left[N\left(\sum Y^{2}\right)-\left(\sum Y\right)^{2}\right]}}
$$




\section{D: Cálculo del coeficiente de correlación}

\begin{tabular}{|ll|c|}
\hline & Correlación de Pearson & $\begin{array}{c}\text { Evaluados/ Si } \\
\text { cumplen }\end{array}$ \\
\hline EVALUADOS/ & Correlación de Pearson & 0.970 \\
CUMPLEN & Sig. (bilateral) & 0.043 \\
& $\mathrm{~N}$ & 3 \\
\hline
\end{tabular}

b Empleando el error típico asintótico basado en la hipótesis nula.

c Basada en la aproximación normal.

\section{E: Decisión}

Como el valor del coeficiente de correlación de Pearson es $r=0.970$ entonces se demuestra que existe un nivel de correlación alta, por lo tanto se rechaza la hipótesis nula $\left(\mathrm{H}_{0}\right)$ y se confirma que existe relación entre la evaluación del transporte público y la contaminación ambiental.

F: $\quad$ Prueba de significatividad de la correlación

Para establecer si el valor del coeficiente es estadísticamente significativo, se aplica el criterio de P_value, donde la regla de decisión es:

$$
\begin{array}{ll}
\text { P_value }<0.05 & \text { Se rechaza la } \mathrm{H}_{0} \\
P_{\text {_ value }}>0.05 & \text { Se acepta la } \mathrm{H}_{0}
\end{array}
$$

Comparando el valor Sig. Aproximada y el valor del nivel de significación, se determina la decisión.

El valor de P_value $0.043<0.05$

Luego con un nivel de significación del 0.05, existe evidencia para rechazar la hipótesis nula $\left(\mathrm{H}_{0}\right)$

\section{G: $\quad$ Conclusión}

Como el valor del estadístico de prueba P-value (0.043) es menor que 0.05 , se concluye, con un nivel de confianza del $95 \%$ que estadísticamente es significativa la evidencia de la existencia de la relación entre las variables evaluación del transporte público con la contaminación ambiental.

\section{CONCLUSIONES}

- $\quad$ El sistema de transporte público influye en la contaminación del aire de los vehículos livianos en la ciudad de Tacna-2014.

- Es limitado el conocimiento que tiene la población sobre el actual sistema de transporte público y la contaminación del aire de los vehículos livianos en la ciudad de Tacna.

- Del análisis de los datos obtenidos con los valores máximos de los estándares de calidad ambiental (ECA) del actual del sistema de transporte público y la contaminación del aire de los vehículos livianos en la ciudad de Tacna en el 2014, se concluye que no se cumplen con todos los estándares de calidad, como es el caso de la partícula PM 2.5 del aire por las emisiones de gases tóxicos. 


\section{REFERENCIAS BIBLIOGRÁFICAS}

- $\quad$ AGUILAR, Segundo (2010). Propuesta para implementar un plan estratégico ambiental para mejorar la calidad del aire en el malecón de atraque al servicio del Perú en Arica 2009.Tesis de Grado de Maestro - UNJBG - Gestión Ambiental y Desarrollo Sostenible.

- $\quad$ ANDIA VALENCIA, Walter (2009) . Manual de Gestión Ambiental, centro de investigación y capacitación empresarial, Perú.

- AVILÉS, Sergio (2014) Apuntes del curso operaciones en transporte, maestría en ingeniería civil con mención en transportes. Escuela de Postgrado de la Universidad Privada de Tacna.

- Banco Central de Reserva del Perú (2013).Informe económico y social región Tacna. Lima. Perú.

- $\quad$ CARTAGENA, Raúl (2008). Valoración económica de la contaminación por el parque automotor y su efecto sobre las viviendas en zonas críticas de la ciudad de Tacna: Año 2008. Tesis de Grado de Maestro - UNJBG - Gestión Ambiental y Desarrollo Sostenible.

- $\quad$ FLORES CANO, José Antonio (2011). Simulación de la contaminación atmosférica y su influencia en el control ambiental de vehículos livianos en Tacna en el periodo 2011. proyecto de tesis para la obtención del grado de maestro- UNJBG - Gestión Ambiental y Desarrollo Sostenible.

- $\quad$ INAPMAS (1997). Contaminación atmosférica y calidad del aire.

- $\quad$ INAPMAS (1997). Legislación Ambiental Peruana.

- $\quad$ MENDOZA, Marisol (2010). Valoración de las Emisiones de Contaminación del Aire Generada por Fuentes Móviles para la Gestión de la Calidad del Aire en el cercado de Tacna.Tesis de Grado de Maestro - UNJBG - Gestión Ambiental y Desarrollo Sostenible.

- $\quad$ Ministerio de Energía y minas (2000).Inventario Nacional Integrado de Emisiones de Gases de Efecto Invernadero del Perú.

- $\quad$ Ministerio de Transportes y Comunicaciones (2001). DS Nº 047-2001-MTC Límites Máximos Permisibles de emisiones contaminantes para vehículos automotores que circulen en la red vial.

- $\quad$ Municipalidad provincial de Tacna (2006).Plan regulador de rutas.

- $\quad$ Organización Mundial de la Salud (2008).Calidad del Aire y Salud.

- $\quad$ PARI, Avelino (2009). Influencia del estado de los vehículos gasolineros y diesel de transporte masivo urbano en el proceso de inspección de gases contaminantes vehiculares en la ciudad de Tacna.

- $\quad$ SALAZAR NAVARRO, Santiago Ramón (2012).La contaminación del aire por la emanación de gases tóxicos producido por vehículos motorizados en lima metropolitana y la función policial.

- $\quad$ TOLCACHIER, Alberto Jorge (2004). Medicina Ambiental. Libro Virtual IntraMed Argentina.

- $\quad$ VISCARRA, Manuel Andreu (1982). La Atmósfera Contaminada y sus Relaciones con el Público.

Recibido: 18/03/2016

Aceptado para publicarse:

4/4/2016 